\title{
Correlates of contraceptive use among HIV discordant couples in Kenya
}

\author{
Kenneth Ngure ${ }^{1,2}$ Zipporah Ng'ang'a ${ }^{2}$, Violet Kimani ${ }^{3}$, \\ Samoel Khamadi ${ }^{4}$, Frankline Onchiri ${ }^{5}$, Elizabeth Irungu ${ }^{1}$,' \\ Renee Heffron', Edwin Were ${ }^{7}$ and Nelly Mugo 1,8
}

\author{
${ }^{1}$ Kenyatta National Hospital, Nairobi, Kenya \\ 2 Institute of Tropical Medicine and Infectious Diseases, Jomo Kenyatta \\ University, Kenya \\ ${ }^{3}$ School of PublicHealth, University of Nairobi, Kenya \\ ${ }^{4}$ Center for Virus Research, Kenya Medical Research Institute, Kenya \\ ${ }^{5}$ Center for Microbiology Research, Kenya Medical Research Institute, \\ Kenya \\ ${ }^{6}$ Department of Epidemiology, University of Washington, Seattle, USA \\ 7 School of Public Health, Moi University, Kenya \\ ${ }^{8}$ Department of Global Health, University of Washington, Seattle, USA
}

Address for correspondence: Kenneth Ngure, Box 19704-00202 Nairobi, Kenya, Phone 254722362219, kngure@pipsthika.org

Despite risks of HIV transmission to infants born of the HIV positive women, contraceptive use is uncommon among women in HIV discordant partnerships. The aim of this study was to determine the factors associated with contraceptive use in a clinical trial cohort of HIV serodiscordant couples based in Thika and Eldoret, Kenya. Data were analyzed from 48I HIV discordant couples enrolled in the Partners in Prevention HSV/HIV Transmission Study at the Thika and Eldoret sites. The primary study outcome was self-reported use of contraception other than condoms. Using a marginal longitudinal logistic model based on generalized estimating equations (GEE) approach we assessed the association of various demographic and behavioral factors with contraceptive use. At baseline the prevalence of non barrier contraceptive use among HIV positive and negative women was $24.3 \%$ and $25.7 \%$, respectively. At month 12 of follow-up, the prevalence of contraceptive use was $44.4 \%$ among the HIV positive and $26 \%$ among the HIV negative women while at month 24 , the prevalence of contraceptive use was $38.6 \%$ among the HIV positive and I $8.2 \%$ among the HIV negative women. HIV positive women were more likely to report using contraception than HIV negative women (odds ratio (OR) $1.6195 \%$ confidence interval (CI) I.04-2.47). Additionally, being married (OR 2.4, 95\% Cl I.2-5.0), attending Thika site clinic (OR 6.I, $95 \% \mathrm{Cl} 4.2-9.0$ ), and having two or more children (OR $1.9,95 \% \mathrm{Cl} 1.3-2.8$ ) were significantly associated with use of non barrier contraceptives. Future programs should focus on interventions to increase contraceptive use among HIV serodiscordant couples, with a special emphasis on HIV negative women, unmarried women and women with few children. 
Keywords: Contraceptive use, HIV, discordant, Kenya, couples, women.

\section{Introduction}

In Kenya about $6.1 \%$ of 1.3 million adults are infected with Human Immunodeficiency Virus (HIV) (KAIS., 2008). However, the contraceptive prevalence rate is low and only $36 \%$ of women report using modern contraceptive methods; there is a $25 \%$ unmet need for contraception among married women (KDHS., 2003). Among married individuals in Kenya who are HIV infected, $45 \%$ have a partner who is not currently infected. In addition 10\% of monogamous married couples and $14 \%$ of polygamous couples are HIV serodiscordant (KAIS., 2008). Numerous reports have demonstrated that stable HIV discordant couples may be responsible for a majority of HIV infections in Africa (Lingappa et al., 2008; Dunkle et al., 2008).

Contraceptive use is the second pillar of prevention of mother to child transmission. This approach involves encouraging sexually active women to avoid unintended pregnancies by using effective methods of contraception. Currently, the main approach to preventing mother to child transmission has primarily focussed on provision of antiretrovirals (ARVs) to mothers and their neonates, even though many HIV positive births could be prevented by preventing unintended pregnancies (O'Reilly.,2003;Wilcher et al., 2008; Reynolds et al., 2008; Cohen.,2008). Among HIV discordant couples, effective contraceptive use has potential to reduce unintended pregnancies and consequently reduce HIV exposed births. Barrier contraceptive methods such as male and female condoms can also prevent HIV transmission between HIV discordant couples.

The limited information regarding the factors that influence contraceptive use among HIV discordant couples in Kenya is a major barrier to developing effective programs for HIV prevention. Such programs can support positive behaviours such as increased contraceptive use and decreased unsafe sexual behaviour. In this study we evaluated the correlates of contraceptive use among HIV discordant couples in a cohort of HIV discordant couples participating in an HIV prevention trial in Kenya.

\section{Methods}

\section{Population and procedures:}

This study was conducted as an ancillary study to the Partners in Prevention HSV/HIV Transmission Study (Lingappa et al., 2009; Celum et al., 2010). Data from 48I HIV discordant couples - 34I in whom the woman was HIV positive and 140 in whom the man was HIV positive - enrolled at the Thika and Eldoret clinical trial sites were analyzed. Partipants were followed for a maximum of 24 months. Enrollment for Thika site began June 2006 and Eldoret site began in November 2004. Follow up for both sites was concluded in October 2008. All HIV positive participants had a CD4>250 cell/ul and were not using ARVs at enrollment. All couples were part of a heterosexual couple and had at least 3 sexual contacts in the 3 months prior to enrollment. During follow up HIV positive women visited the study clinic monthly while the HIV negative women visited the clinic quar- 
terly.

For this analysis, the primary outcome measure was self-reported use of contraception other than condoms. This was defined as current use of an intra uterine device (IUD), surgical methods, injectable, implantable, or oral hormonal methods. Contraceptive use was compared between HIV positive and HIV negative women during the 24 month follow-up period.

\section{Statistical Analysis}

The collected data were cleaned for errors and inconsistent answers. To describe the characteristics of the study participants, the t-test was used to compare differences in the means of continuous variables and chi-square testing was used to compare differences in categorical factors. To model the population-average likelihood of contraceptive use a longitudinal logistic model for the marginal probability of contraceptive use at each time point during follow-up was fitted using a generalized estimating equations (GEE approach). The probability of contraceptive use was modelled as a logistic function of the covariates thought to be predictive of contraceptive use. A covariate-adjustment for other potentially confounding variables was done to allow for the analyses of their possible role on the association between contraceptive use and predictors of contraceptive use and changes in the likelihood of contraceptive use over the time. The visit month was modelled as a quadratic variable. All the data analyses were done using STATA v.II.0 (Stata Corp; College Station, TX). We used two-sided tests with $p$-values of $<0.05$ being considered to be statistically significant.
Ethical considerations:

This analysis was approved by the Kenya Medical Research Institute (KEMRI) Ethical and Scientific review Committees and the Moi University Institutional Research and Ethics Committee (IREC).

\section{Results}

Characteristics of participants

Data from 48I HIV discordant couples were analyzed. The HIV positive and negative women were statistically similar with respect to almost all the selected baseline characteristics. The baseline characteristics of the study participants are summarized by their HIV status in Table I.

\section{Contraceptive use}

The baseline prevalence of contraception was $24.3 \%$ for HIV positive women ( $19.8 \%$ among the HIV positive women in Eldoret and $29.6 \%$ among HIV positive women in Thika). The baseline prevalence was $25.7 \%$ for HIV negative women (18.6\% among HIV negative women at Eldoret site and $37 \%$ among HIV negative women in Thika site). At each follow-up visit, the prevalence of contraceptive use was higher among the HIV positive patients at each month relative to the HIV negative group (Figure I). There was consistent increase in proportion of participants using contraception until month 18 among the HIV positive women when contraceptive use started to decline. Among the HIV negative women, contraceptive use tends to be nearly constant across time and starts to decline by month 18 of follow-up. Notably, most participants were exited the Partners in Prevention study before 
completing their 24 months of follow

end.

up as a result of the study coming to an

Table I Baseline Characteristics of HIV-I positive and negative women

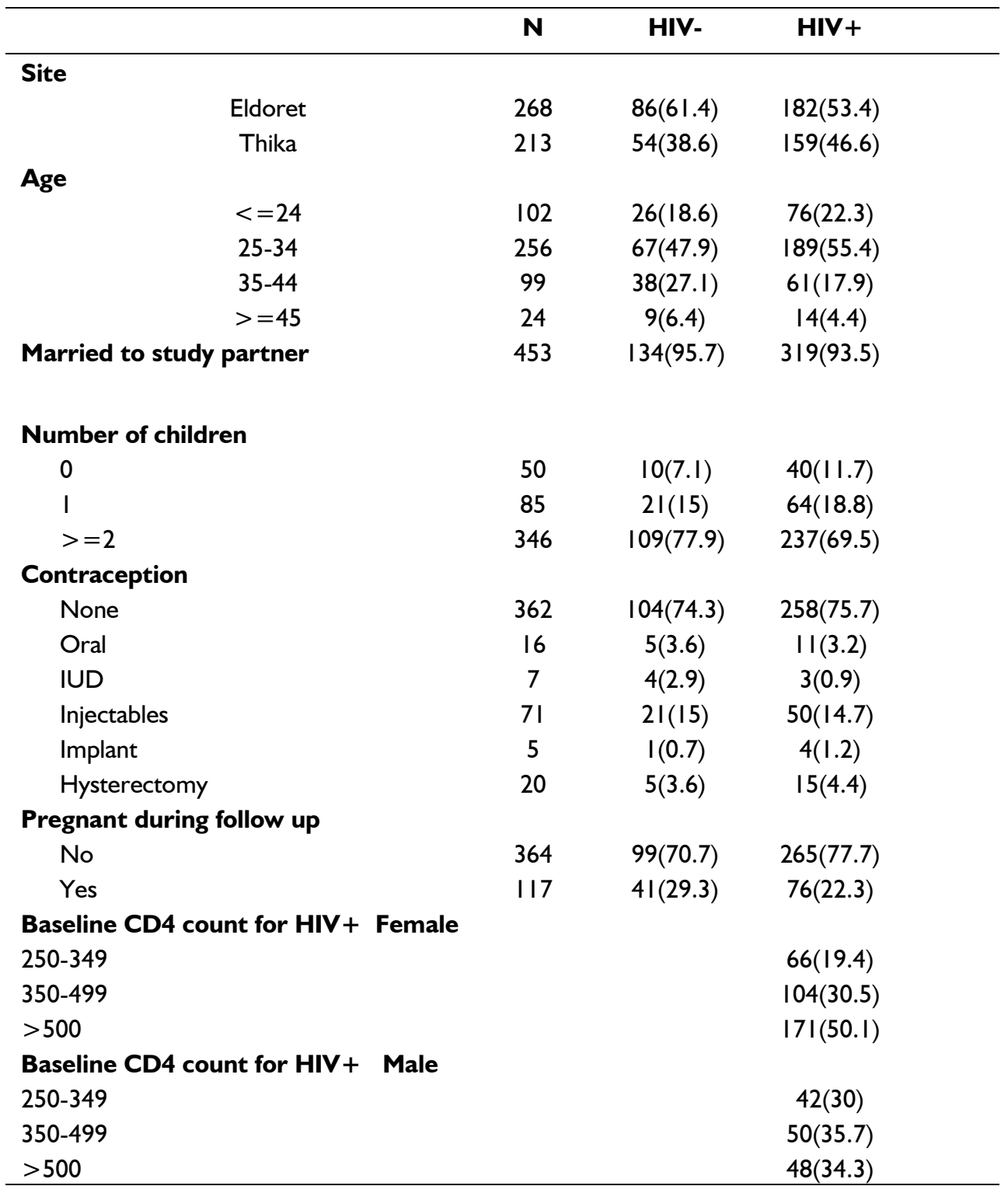

The proportion of participants using various forms of non-condom contraceptives at any given month during follow-up is shown in Table 2. We observed that at any follow-up month, injectable methods were the most common, while almost over $60 \%$ of

participants were not using any form of contraception.

Reported condom use was high through out the follow up period for sex with study and non study partners. This rose from $75.47 \%$ at baseline to $94.02 \%$ at month 24 of follow up for 90 
sex with study partners and remained with other partners.

$>95 \%$ through out follow up for sex

Table 2 Contraceptive methods used during study visit months as among HIV positive and HIV negative women

\begin{tabular}{ccccccccccc}
\hline & \multicolumn{10}{c}{ Collow-up Month } \\
\hline Contraception & 0 & 3 & 6 & 9 & 12 & 15 & 18 & 21 & 24 & Total \\
\hline $\mathrm{N}$ & $48 \mathrm{I}$ & 463 & 450 & 427 & 397 & 348 & 263 & 156 & $1 \mathrm{II}$ & \\
\hline None & 75.26 & 75.50 & 70.07 & 65.24 & 63.01 & 59.02 & 59.31 & 65.13 & 67.39 & 67.13 \\
Oral & 3.33 & 1.99 & 2.49 & 3.81 & 5.25 & 6.34 & 6.30 & 5.46 & 4.35 & 4.21 \\
IUD & 1.46 & 1.10 & 1.13 & 0.95 & 0.72 & 0.98 & 0.86 & 1.68 & 0.54 & 1.06 \\
Injectables & 14.76 & 15.23 & 18.59 & 21.67 & 21.00 & 22.44 & 22.06 & 19.75 & 21.74 & 19.35 \\
Implant & 1.04 & 1.55 & 2.27 & 2.86 & 3.82 & 4.88 & 5.44 & 4.20 & 2.72 & 3.06 \\
Hysterectomy & 4.16 & 4.64 & 5.44 & 5.48 & 6.21 & 6.34 & 6.02 & 3.78 & 3.26 & 5.18 \\
\hline
\end{tabular}

Table 3 Marginal logistic regression analysis of baseline HIV status and longitudinal contraceptive use using generalized estimating equations
Covariate
$\mathrm{N}$
OR
$\mathrm{P}$

$95 \% \mathrm{Cl}$

HIV status at enrolment

Negative*
Positive

Visit month

Married to study partner

No*

Yes

Age

Site

Eldoret*

Thika

Pregnant during follow up

$$
\begin{aligned}
& \text { No* } \\
& \text { Yes }
\end{aligned}
$$

Number of Children

$\begin{array}{ccccc}\text { I* } & 809 & \text { I } & & \\ >=2 & 2286 & 1.868 & 0.003 & (1.245-2.804)\end{array}$

\section{Correlates of contraceptive use}

The odds of contraceptive use were I.6I (95\% Cl: (1.04-2.47) times as likely among the HIV positive women than in those who were HIV negative (Table 3). From the model further sta- tistically significant correlates of non barrier contraceptive use were being married $(\mathrm{OR}=2.4$, 95\% Cl I.2-5.0), coming from Thika site $(\mathrm{OR}=6.1$, $95 \% \mathrm{Cl} 4.2-9.0)$ and having two or more children $(\mathrm{OR}=1.9,95 \% \mathrm{Cl}$ I.3- 
2.8). Being on $A R V s$ and the $C D 4$ count of the HIV positive partner were not significantly associated with contraceptive use. Also not associated with contraceptive use was whether or not both male partner used condoms, whether or not both male and female earned income, whether or not male or female has been abused, years of education for both male and female and female being pregnant during follow-up (data not shown).

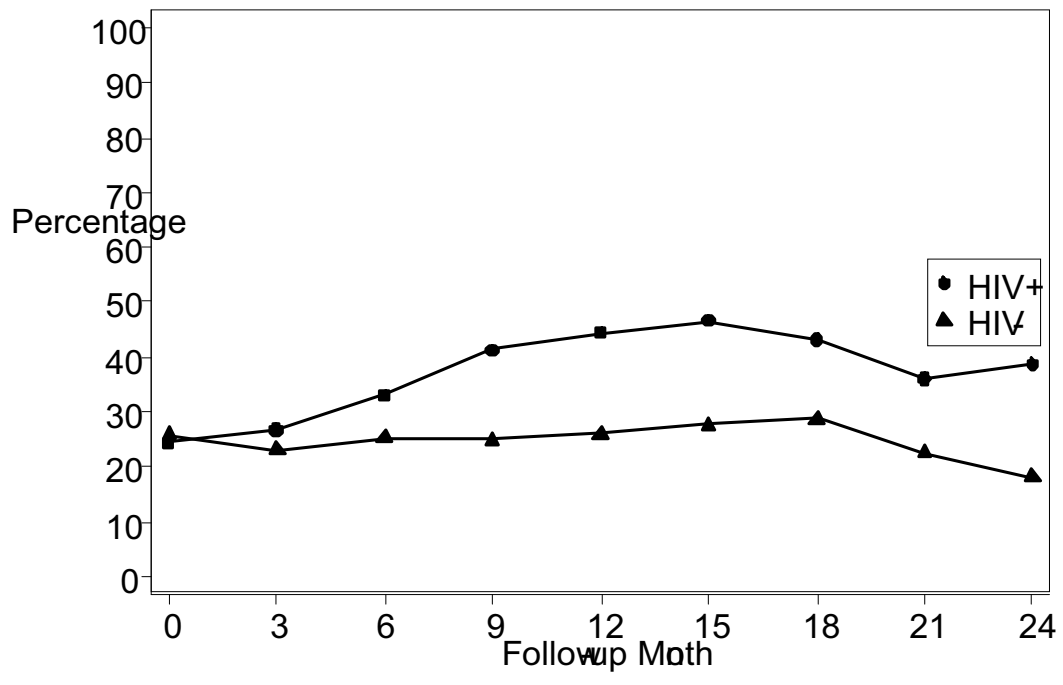

Figure 1: Prevalence of contraceptive use during follow -up

\section{Discussion}

This prospective study evaluated the correlates of contraceptive use among HIV discordant couples in an HIV prevention trial setting in Kenya. HIV positive women were more likely to report contraceptive use during follow up visits than HIV negative women. This result is similar to other studies conducted in resources poor countries that have found that HIV status may strongly influence contraceptive use, with HIV positive women reporting a significantly higher use (Batya et al., 2009; Hoffman et al., 2008; Keogh et al., 2009). However, this finding contrasts the (KAIS.,
2007), which reported higher contraceptive use among HIV negative women compared to the HIV positive women. One possible explanation is HIV positive women in our cohort attended the clinic on monthly basis and thus received more contraceptive counselling compared to HIV negative women who attended the clinic on a quarterly basis.

We observed that being married was significantly associated with an increased likelihood of using contraceptives. Similar findings were reported among HIV positive women receiving prevention of mother to child transmis- 
sion (PMTCT) services in Rwanda and Cote d'lvoire where women in a union were more likely to report a modern method (Batya et al., 2009;Desgrees et al., 2002).

In this analysis, having more children was significantly associated with increased likelihood of using contraceptives. This has been reported in a study among HIV positive and negative PMTCT clients in a South Africa where having fewer children was associated with greater pregnancy desire among the HIV negative women (Peltzer et al., 2009). Additionally, a study among HIV positive and negative women in the national PMTCT program in Rwanda found women with fewer children had a stronger desire for additional children (Hoffman et al., 2008).

Thika site had a significantly higher contraceptive use than Eldoret site perhaps due to the successful multipronged family planning strategy that was invoked at the Thika site and not in Eldoret. This strategy involved training of counselors and clinicians and provision of non-barrier contraceptive methods free of charge at the research clinic (Ngure et al., 2009).

Over half of the contraceptive users chose injectables compared to other forms of contraceptives at each timepoint. This reflects the pattern in many African countries where the Injectable contraceptive depot-medroxyprogesterone acetate (DMPA) is a popular option among women, due to among other reasons ease of use and the opportunity for discreet use (Lavreys et al., 2004).

The strengths of our study include its design as a prospective cohort study and its ability to observe contraceptive use over time and up-to 24 months of follow-up for each couple. As both members of the couple were enrolled in the study, we were able to assess contraceptive use among HIV positive and HIV negative women and include data on male HIV positive partners to accompany data on the behaviors of HIV negative women. A lack of information on male partners has been a limitation in many contraceptive studies (Wilson et al., 2003). Our study is a sub-analysis within the larger prospective study of contraceptive use among over 3400 African women in HIV-I serodiscordant partnerships (Heffron et al., 2010). We focused our sub-analysis specifically on contraceptive use in 2 different geographical regions in Kenya and this enabled us to evaluate the effect of multiple and additional variables on contraceptive use.

Our study had a number of limitations. First this study was carried out in the context of a clinical trial setting, where contraception was mainly provided free of charge at one of the study clinics (Thika). This may reduce the generalizability of the findings to the general Kenyan population. Secondly, our study relied on self reported contraceptive use and we did not validate its accuracy. Thirdly, we did not assess pregnancy intention and fertility desires in this study and this limits our ability to interpret the results. Lastly Thika and Eldoret sites were among the last sites to be activated in the Partners in Prevention trial, therefore by the time the study came to an end most of the participants had not completed their 24 month follow-up, perhaps a more consistent trend would have been observed with a longer follow-up. 
In conclusion, promoting dual contraceptive use among HIV discordant couples is of critical importance. Future programs should focus on interventions to increase non-barrier contraceptive use among women in serodiscordant relationships, with a special emphasis on single women with few children and their male partners using a multipronged pronged strategy (Ngure et al., 2009).

\section{Acknowledgements}

We thank the clinical trial staff at the Thika and Eldoret sites of the Partners in Prevention HSV/HIV Transmission Study for use of site data for this analysis. The Partners in Prevention study was funded by the Bill and Melinda Gates foundation \# 26469. This analysis was supported by the African Doctoral Dissertation Research Fellowship offered by the African Population and Health Research Center (APHRC) in Partnership with the International Development Research Center (IDRC) and Ford Foundation. Preliminary analyses from this study were presented at the International AIDS Conference (IAS 2010) poster \# I4 I65

\section{References}

Celum, C., Wald, A., Lingappa, J.R., Magaret, A.S., Wang, R.S., Mugo, N., et al. (2010) Acyclovir and Transmission of HIV-I from Persons Infected with HIV-I and HSV-2. N Engl J Med 362: 427-439.

Central Bureau of Statistics (CBS) (Kenya), Ministry of Health (MOH) (Kenya), \& ORC Macro. (2003) Kenya Demographic and Health Survey. Calverton, Maryland: CBS,
$\mathrm{MOH}$ and ORC Macro; 2004.

Desgrees du Lou, A., Msellati, P., Viho, I., Yao, A., Yapi,D., Kassi, P., et al.(2002) Contraceptive Use, Protected Sexual Intercourse and Incidence of Pregnancies among African HIV-infected Women. International Journal of STD \& AIDS 13(7): 462468.

Dunkle, K.L., Stephenson, R., Karita, E., Chomba, E., Kayitenkore, K., Vwalika, C., et al.( 2008) New heterosexually transmitted HIV infections in married or cohabiting couples in urban Zambia and Rwanda: an analysis of survey and clinical data. Lancet; 37I: 2183-2।91

Elul, B., Delvaux, T., Munyana, E., Lahuerta, M., Horowitz, D., Ndagije, F., et al. (2009) Pregnancy desires, and contraceptive knowledge and use among prevention of mother-to-child transmission clients in Rwanda. AIDS Vol. 23, No. Suppl I: S19-S26

Heffron, R., Were E., Celum, C., Mugo, N., Ngure, K., Kiarie, J., et al.(20l0) A Prospective Study of Contraceptive Use among African Women in HIV-I Serodiscordant Partnerships. Sex Transm Dis 68(7):4207-42I6.

Hoffman, I.F., Martinson, F.E., Powers, K.A., Chilongozi, D.A., Msiska, E.D., Kachipapa, E.I., et al. (2008). The year-long effect of HIV-positive test results on pregnancy intentions, contraceptive use, and pregnancy incidence among Malawian women. J Acquir Immune Defic Syndr. I;47(4):477-83.

Keogh, S. C., Urassa, M.,Kumogola, Y., Mngara, J., Zaba, B., (2009) Reproductive behaviour and HIV status of antenatal clients in northern Tanza- 
nia: opportunities for family planning and preventing mother-to-child transmission integration. AIDS Vol. 23, No. Suppl I: S27-S35

National AIDS and STI Control Program (NASCOP), Ministry of Health (MOH). (2007) Kenya. Kenya AIDS Indicator Survey: Preliminary Report. Nairobi, Kenya: Republic of Kenya; 2008.

Ngure, K., Heffron, R., Mugo, N., Irungu, E., Celum, C., Baeten, J., (2009) Successful increase in contraceptive uptake among Kenyan HIVI-serodiscordant couples enrolled in an HIV-I prevention trial. AIDS Vol. 23, No. Suppl I: S89-S95

O'Reilly, K. (2003). Preventing HIV in infants and young children. PMTCT and integration. Presented at Reproductive Health in the Age of HIV/ AIDS, San Juan, Puerto Rico.

Lingappa, J.R., Lambdin, B., Bukusi, E.A., Ngure, K., Kavuma, L., Inambao, M., et al.( 2008). Regional differences in prevalence of HIV-I discordance in Africa and enrollment of HIV-I discordant couples into an HIV-I prevention trial. PLOS ONE; 3: el4II.

Lingappa, J.R., Kahle, E., Mugo, N., Mujugira, A., Magaret, A., Baeten, J., et al. (2009). Characteristics of HIVI discordant couples enrolled in a trial of HSV-2 suppression to reduce HIV transmission: The Partners
Study. PLoS ONE; 4: e5272.

Peltzer, K., Chao, L.-W. \& Dana, P. (2009) Family planning among HIV positive and negative prevention of mother to child transmission (PMTCT) clients in a resource poor setting in South Africa. AIDS and Behavior. 13:973-979

Reynolds, H.W., Janowitz, B., Wilcher, R., Cates, Jr W., (2008). Contraception to prevent HIV-positive births: current contribution and potential cost-savings in PEPFAR countries. Sex Transm Inf; 84 (Suppl 2): ii49-5.

Cohen, S. A., (2008) Hiding in Plain Sight: The Role of Contraception in Preventing HIV, Guttmacher Policy Review Winter, Volume II, Number I.

Westoff, C.F, Bankole A.,(2000) Trends in demand for family limitation in developing countries. Int Fam Plann Perspect;26:56-62

Wilcher, R., Petruney, T., Reynolds H.W., Cates W., (2008) From effectiveness to impact: contraception as an HIV prevention intervention. Sex Transm Inf; 84 (Suppl 2): ii54-ii60.

Wilson., T. E., Koenig, L., Ickovics, J., Walter, E., Suss, A.,; Fernandez, M. I., (2003) Contraception Use, Family Planning, and Unprotected Sex: Few Differences Among HIVInfected and Uninfected Postpartum Women in Four US States JAIDS Volume 33, 5 pp 608-613. 